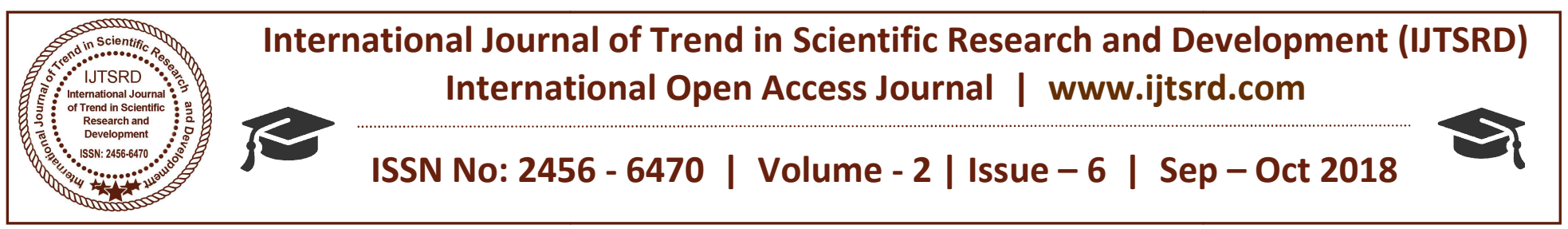

\title{
Impact of Early Physiotherapy of Paraplegic Patients
}

\author{
Dr. Laxmi Barve, Dr. Madhuri Paikera, Dr. Shubhra Dubey \\ Physiotherapist, Chhattisgarh, Raipur, India
}

\begin{abstract}
The physiotherapy program primarily focused not only on the rehabilitation of the patients, but also to improve their quality of life. The treatment phase varied in cases with paraplegia and tetraplegia from 8 (average days: 62) to 12 weeks (average days: 73), respectively. The physiotherapists followed some key steps to formulate the process of physiotherapy to achieve highest rate of success with minimized failures. A comprehensive and effective physiotherapy program is required for the complete rehabilitation of the patient which involves goal planning. The goals were articulated by the patient as per their requirement and were finalized by the multidisciplinary team including clinicians and physiotherapists.
\end{abstract}

Keyword: early physiotherapy, spinal cord injury, paraplegia

\section{Background:-}

Physiotherapy techniques in paraplegia have developed considerably in the last ten years since the proper establishment of spinal units. The paraplegic patient is best served by such units where the patientphysiotherapist relationship is indeed a close one. All the techniques of physiotherapy have one aim in view, the complete rehabilitation of the patient.

In the management of the paraplegic patient the role of the physiotherapist, as one member of the rehabilitation team, includes aid in the prevention of deformities, treatment of such deformities as occur, training in ambulation and in the performance of all the routine activities of daily living, the provision of useful tasks for the unaffected arm, the development of maximum usefulness in the affected limbs, and assistance in speech training when indicated.

Spinal cord injury (SCI) is defined as the incidence of temporary or permanent sensory and/or motor deficiency due to occurrence of an acute abrasion of the neural elements present in the spinal canal, i.e., spinal cord and cauda equine. Depending upon the severity and location of injury on the spinal cord, the patients confront partial or complete loss of sensory, autonomic and motor functions below the level of injury. The incomplete and complete loss of sensory and/or motor functions injury found below the neurological level, including the lowest sacral segment, is referred to as an incomplete and complete injury, respectively.

Every year, SCI degrades the quality of life of around 40-80 people per million population around the world among adults, male-to-female ratio of at least $2: 1$ is reported. Mortality risk is about 2-5 times higher in people with SCI as compared to the unaffected people As per the report on the causes of SCI from the National Spinal Cord Injury Statistical Center, vehicle accidents $(39.08 \%)$ are the most common cause of SCI followed by falls (29.54\%), acts of violence $(14.41 \%)$, sports and recreational activities $(8.39 \%)$ and other causes $(8.57 \%)$ that include medical surgical complications, pedestrian, hit by falling/flying object.

Based on the level of injury, patients with SCI are referred as tetraplegics (quadriplegics) or paraplegics. Tetraplegia refers to a damage to the cervical spinal cord segments at levels $\mathrm{C} 1-\mathrm{C} 8$, whereas, injury to brachial plexus lesions is not included. Paraplegia includes injury to caudaequina and conusmedullaris, thoracic vertebrae (T-1 to T-8 and T9 to T12), lumbar and sacral regions of the spinal column, but excludes injury to the lumbosacral plexus lesions Injury to the peripheral nerves outside the neural canal is not included in any of them It has been reported that since 2005 , about $40.85,21.6,21.4$ and $15.8 \%$ of the patients with neurological disorders were affected with incomplete tetraplegia, complete paraplegia, 
incomplete paraplegia and complete tetraplegia, respectively

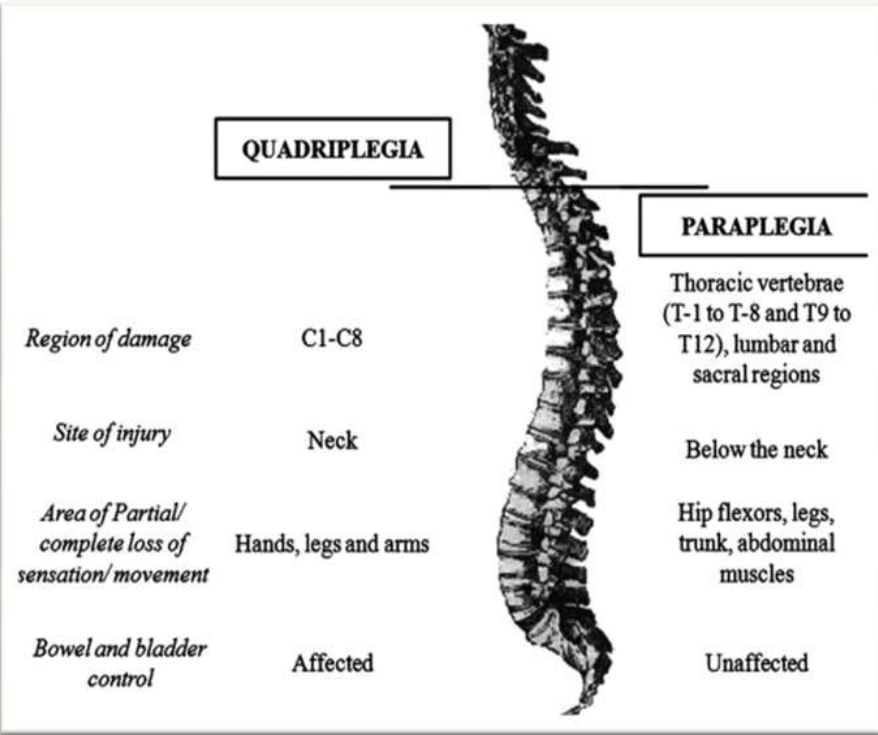

\section{Tetraplegia and paraplegia}

Management therapies attributed to tetraplegia and paraplegia involve the use of steroids, pain medications, surgery, bed rest, etc. Stem cell therapy using neural stem cells (NSCs), adult stem cells such as mesenchymal stem cells (MSCs), etc. has been investigated as a promising tool for the treatment of SCI Human embryonic stem cells (hESCs) have a huge potential of self renewal, pluripotency and unlimited propagation In previous studies, the beneficial effects of hESC therapy have been reported in patients with chronic SCI.

Physiotherapy helps individuals with SCI to be able to function with their injuries in a day to day situation. It involves exercising for mobilization as well as for stimulation of the nerves and muscles below the level of the injury besides, it may also help in restoring the functioning of atrophied muscles Though largely helpful, it only helps the patients with SCI to live with their injury and to prevent further deterioration. The interdisciplinary management of SCI involves hESC treatment as well as physical therapy (as supportive therapy) for rehabilitation of the patients with SCI. The twin-benefit, i.e. the retraining of the muscles during physiotherapy as well as the regeneration of the spinal cord due to hESC, is the likely reason that the patients have shown benefits in their clinical condition the present study focuses on the role of physiotherapy in the mobilization of patients with SCI receiving $\mathrm{hESC}$ therapy.
Abbreviations

SCI spinal cord injury

NSCs neural stem cells

hESCs human embryonic stem cells

AIS American Spinal Injury Association Impairment Scale

ROM range of motion

THKAFO trunk-hip-knee-ankle-foot orthosis

KAFO knee-ankle-foot orthosis

AFO ankle-foot orthosis

HKAFO hip-knee-ankle-foot orthosis

MRI magnetic resonance imaging

ISO International Standards Organization

\section{Introduction:-}

The patients undergoing hESC therapy started taking physical therapy as a support therapy. It included various exercises, gait training as well the use of devices for positioning of joints and the mobility aids.

\section{Exercises}

Exercise is important for patients with SCI. A wide range of exercises were practiced by paraplegics and tetraplegics depending upon the level of injury.

\section{Range of motion (ROM) exercises:}

Patients with SCI at T10 and above are usually ambulated for . ROM exercises are of two types, i.e., active and passive. Active (self) ROM exercises included the exercises performed by the patient himself such as stretching; the patient uses his strong arm that helps the weaker arm to perform exercises. Studies report the use of stretching as an effective way to treat and prevent contractures. Passive ROM exercises needed the assistance of a physiotherapist. They were performed within the ROM to avoid fatigue, swelling and pain. Though, they did not strengthen the muscles, but, helped in avoiding stiffness of joints and maintained functional capacity

ROM exercises helped the patients in preservation as well as improvement in the flexibility and mobility of the joints. They also reduced the stiffness and risk of freezing of joints during the progression of disease.

\section{Isometric, active or active-assisted truncal exercises:}

The patients with partial movement in joints/incomplete injury were made to perform these exercises for early mobilization. Recent studies have also shown that early mobilization plays an important 
role in the prevention of pulmonary function decline and aid in muscle strengthen in

\section{Empowering exercises:}

Empowering exercises are the active and resistance exercises which were performed by patients with SCI to restore the strength of upper extremities and shoulder rotation required for swimming, using electric bicycles and walking

\section{Weight and resistance exercises:}

The muscle strength of paraplegics and tetraplegics was improved by weight and resistance exercises. These exercises were performed using dumbbells in bed, the weight of which is dependent on the muscle strength of the patient

In addition, breathing exercises were also performed by complete or incomplete paraplegic and tetraplegic patients to protect their lung capacity and strengthen their upper extremities. Generally, tetraplegics suffer from impaired chest wall and diaphragm muscle function which leads to an improper functioning of the lungs, subsequently causing death. Therefore, regular airway clearance therapy was essential

\section{Gait training:}

Gait training is provided to patients with SCI to encourage them to stand and walk where ever possible. Gait cycle is divided into two phases, i.e. stance and swing. The ratio of time consumption by each phase is $3: 2$, where stance and swing occurs when the foot is on and off the ground, respectivel

\section{Use of parallel bars:}

People with an "incomplete" SCI, irrespective of the level of injury, have more potential to regain walking than those with a "complete" SCI. Therefore, parallel bars were used for ambulation to achieve truncal and pelvic stabilization in the patients. Once the patients were able to stand upright, either with/without a posterior shell, a blend of standing, balancing and moving exercises were performed with the support of a posterior shell in the parallel bars and a long-locked knee joint walking device to ensure the integrity and stability of the lower extremity joints in patients

\section{Mobility aids:}

The patients with SCI are usually asked to use walkers or wheelchairs for mobility. Depending upon the need and severity of injury, the wheelchairs were customized in terms of dimensions such as the height, pelvic width, seat length, backrest, seat and arm support.

The customization of wheelchairs (as per patientes requirement) allowed optimal mobility of the patients, protection of their skin integrity and maintenance of normal anatomical posture. In cases with affected upper extremities, a battery assisted wheelchair was used, whereas, a manual wheelchair was preferred for the patients with injury at lower levels. However, wheelchairs lead to secondary complications due to long term sitting A modification of wheelchair, i.e., TEK robotic mobilization device is a mobility aid which offers a significant improvement in mobilization over wheelchair.

Walkers or walking frames are considered more stable than other mobility aids because of their large base. Their center of gravity also falls within the base The walkers were also designed, keeping in mind, the level and severity of injury. represents a wide walker with an elbow support. Such walking frames were recommended to the tetraplegic patients. For patients who had lost hand grip, the hands could be tied with a strap to the walker. These straps could be removed on improvement of the hand grip.
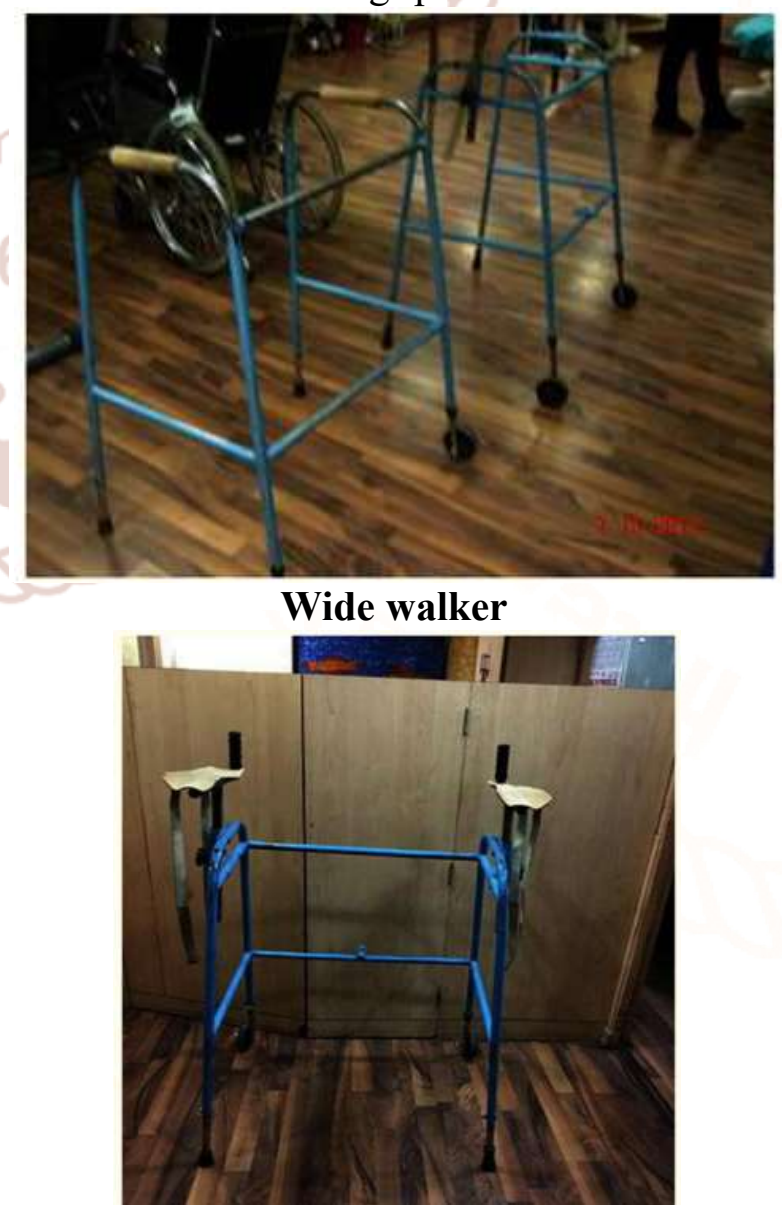

Wide walkers with an elbow support 


\section{Positioning of the joints:}

Positioning of the joints plays an important role in the mobilization of the patients with SCI. In order to protect the articulary structure and maintain the optimal muscle tonus; sand bags, pillows, plaster splints or more rigid orthotic devices were used The patients were prescribed with orthosis, depending upon the severity of injury, the extent of deformities, contractures and stiffness. An improvement in the patients (after hESC therapy and physiotherapy) led to the modification/reduction in the orthotic devices. The tetraplegics were recommended with trunk-hip-kneeankle-foot orthosis (THKAFO). THKAFOs are prepared by attaching a thoracic extension to kneeankle-foot orthosis (KAFO). They help the patients in controlling the hip region as well as in supporting the trunk and the lower limbs. As the hESC treatment progressed, an improvement was observed in the patients with tetraplegia which led to a change in the type of caliper. The caliper was reduced to hip-kneeankle-foot orthosis (HKAFO; The further improvement, in terms of stability and balancing, observed in patients reduced the caliper to a KAFO with a Shannon"s brace. Shannon"s brace is a customized waist belt tied onto the thoraco-lumbar region. It has been named after the first patient who used it . Strengthening of the thoraco-lumbar region was the major advantage associated with the use of Shannon"s brace as it did not provide support to the pelvis portion of the patient. Subsequently, the patients were able to walk without brace. Further improvement in the patients led to a reduction to knee extension brace with an ankle-foot orthosis (AFO) followed by Enrique brace. Enrique brace is a gentler/simpler knee brace which is also named after the patient who used it for the first time. This knee brace can rotate at the level of knees and gives more flexibility to the patients.

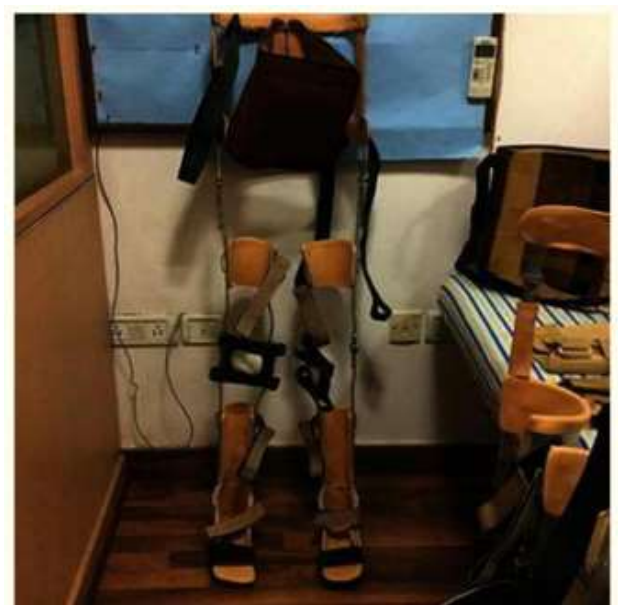

Thoracic hip knee ankle foot orthosis (THKAFO)

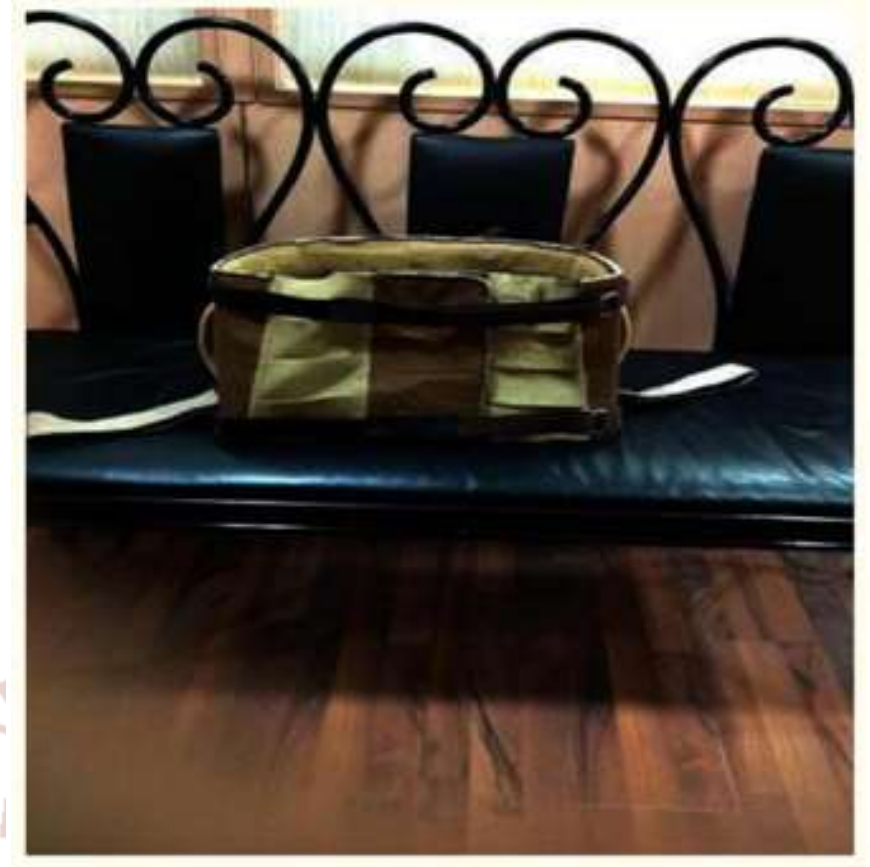

Hip knee ankle foot orthosis (HKAFO)

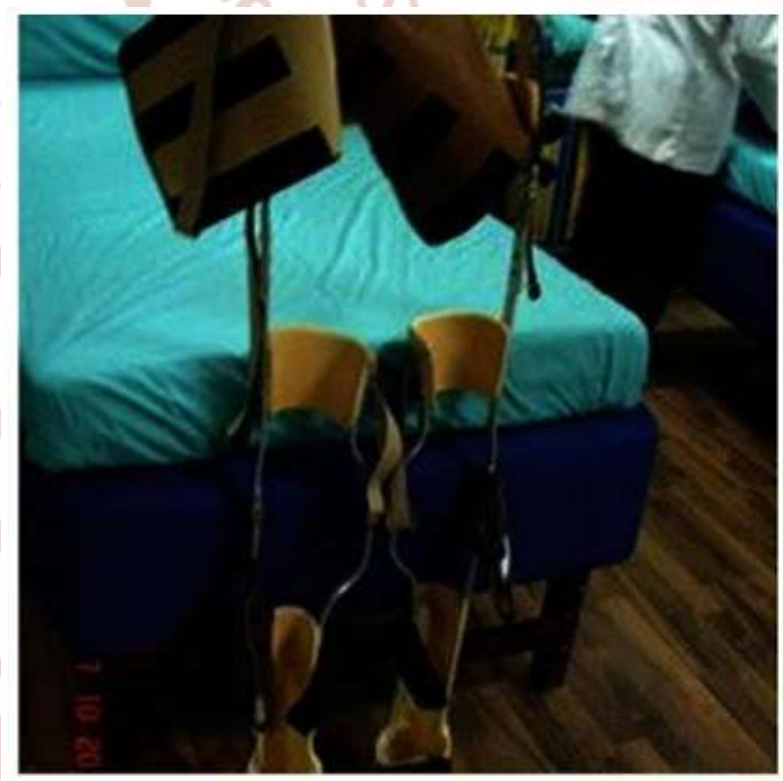

Shannon's brace

The patients with paraplegia were made to stand with full support on a HKAFO. The orthotic devices were modified and designed depending upon the condition and requirement of the patients. The paraplegics were assessed on similar lines as tetraplegics. As connectivity was regained, the support was reduced to KAFO followed by a knee brace and an ankle support. Subsequently, upon improvement, the patients just used ankle support for standing and walking. The walking aids also reduced from walkers along with manual support to just a walker. The patients showed improvement which reduced the orthotic devices to crutches further, only walking sticks were used and finally no aid was needed. 


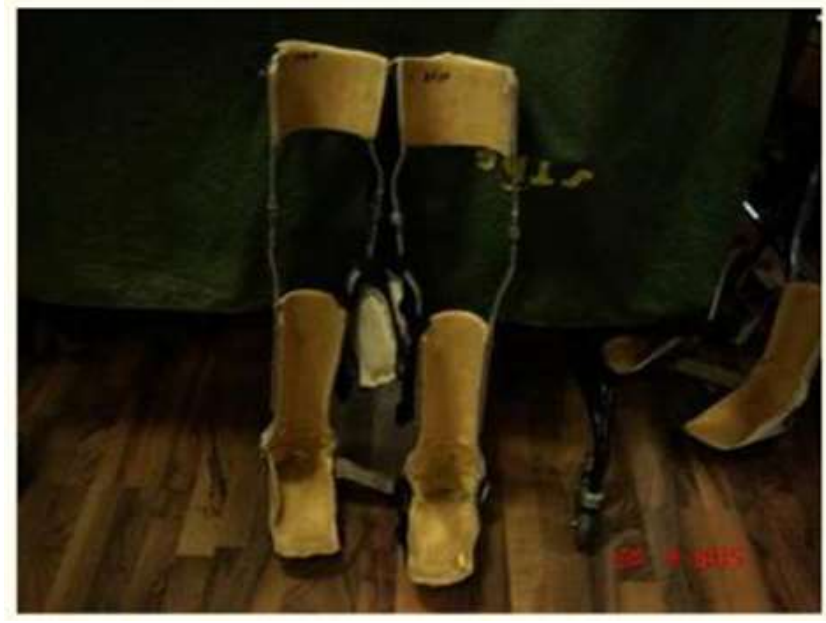

Knee-ankle-foot orthosis (KAFO)

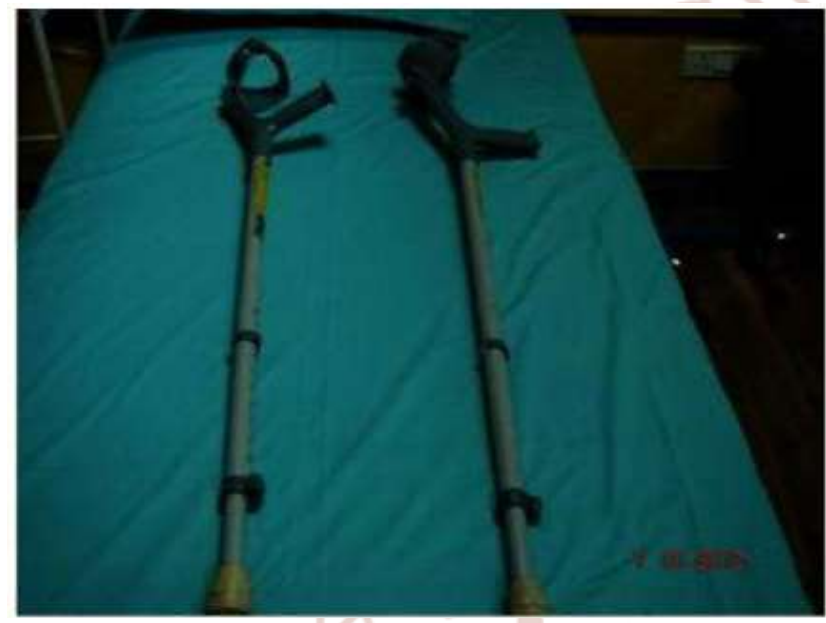

Elbow crutch

Tetraplegics as well as paraplegics initiated their journey of standing and walking with the help of a walker. They were further reduced to wide walkers. Subsequently, they were replaced by normal walkers. When the patients improved further, they were able to walk with the help of a stick.

After every 4-6 months, (at each level), the patients were individually examined to assess the level of improvement, on the basis of which calipers were reduced. They were designed and customized depending upon the level of deformity and pathological condition of the patient. Different materials were used for the preparation of the orthotics which included aluminium, aluminum alloy, thermosetting plastic, iron, leather, rubber, foam, velcro, silicon, etc.

\section{Review of Literature:-}

1. The medical condition where the person experiences loss of feeling or movement in the lower half of the body is known as paraplegia. It is also described as a spinal cord injury or partial paralysis in which the functions of the body waist down are impaired. Injury or disease caused to the spinal cord or brain or both are amongst the primary paraplegia causes. When paraplegia strikes, the brain or spinal cord is unable to send or receive signals to the lower half of the body.

2. Due to this, paraplegics cannot move their body parts below the waist but lose sensation in that area too. At times there is a tingling feeling too in the legs and feet. As a result of some injuries, temporary paralysis in one or both legs can also be caused. It will take a doctor anything between a few hours to a couple of days to diagnose the patient"s condition accurately.

3. The paraplegic patent often has been dismissed as one of the unfortunate by products of war or accident and thereby often has been the victim of neglect and ignorance . the large number of persons with paraplegia resulting from world war injuries necessitated establishment of paraplegia centers where patient rehabilitated as fully as possible. it soon become evident become a primary problems in these long term patients were directly related to metabolic changes caused by their condition. Many of these changes caused by their condition. Many of these changes had been noted in paraplegic patient in civilian life, but the small opportunity for study of large group of patient often prevented the development of thought and investigation of these problems

4. one of the most obvious benefits of a robotic exoskeleton is that it allows paraplegics to walk again since they have lost the brain function to do so. As a result, being able to walk again can be a hugs stress relief for both the individual and their family . according to the Christopher and Dana reeve foundation ,individual who are paralyzed are two to three times as likely to develop depression as the non-disable the highest risk of suicide occurs between the first five years of paralysis diagnosis .caregiver depression is also a common illness that can arise from the stress of talking care of a paraplegic

5. Preface Spinal cord injury causes a host of physical and psychosocial problems that can interfere with an individual's health, feelings of well-being, and participation in activities and relationships within the family and community. The goal of rehabilitation after spinal cord injury is to enable the person to resume and then continue a lifestyle that is healthy, fulfilling, and 
integrated with his or her family and community. Physical therapists play a central role in this process, working with recently injured people and their families to maximize physical capabilities and mobility, and to develop the knowledge and skills needed to remain healthy. Three major elements are required for a therapist to fulfill this role most effectively.

6. The Spain Rehabilitation Center is nationally recognized as one of the Southeast ${ }^{\text {ec }}$ foremost providers of comprehensive rehabilitation care for Spinal Cord Injuries and Traumatic Brain Injuries, and The Spinal Cord Injury Center is one of 12 Model Systems in the country.
$>$ Stroke.

$>$ Hereditary spastic paraplegia, which is a genetic disorder.

$>$ It can be congenital, i.e. present at birth.

$>$ Presence of tumor within the spinal cord or constricting the spinal cord.

$>$ Autoimmune diseases.

$>$ Spinal cord disorders, such as syrinx.

$>$ Infection.

9. Spinal Cordlinjury ( $\mathrm{SCI}$ ) occurs when the spinal cord gets damaged following an accident, falls, sporting injuries, or following diseases, such as Polio, Spinal Bifida or Transverse Myelitis.
Rehabilitation Nursingec role includes those of caregiver, patient/family educator, counselor, care coordinator/manager, advocate, consultant, researcher and administrator/manager. At Spain Rehabilitation Center we encourage our nurses to become certified in Rehabilitation Nursing to validate best practice. Nurses and patient care staff work with a multidisciplinary team to serve patients. This team often includes physician, advanced practice nurses, nurses, respiratory therapists, patience care technicians, radiology technicians, dieticians, chaplains, and numerous therapy professionals.

$>$ Primary Spinal Cord Injury and Amputee Patients

> Stroke, multiple trauma, neurological disorders, traumatic brain injury, and cancer-related diagnosis

$>$ Adult population, adolescents, and geriatrics

7. physical therapy exercise are based on a detailed understanding of how the body work - posture, balance and movement, knowledge of diseases, injury and the healing process physiotherapy is a health care profession that aims to develop, maintain and restore maximum functional ability throughout life.

8. Paraplegia, sometime called partial paralysis, is a form of paralysis in which functionis substantially impeded from the waist down. Most people with paraplegia have perfectly healthy legs. Instead, the problem resides in either the brain or the signals card, which cannot send or receive signals to the lower body due to an injury or disease.

$>$ Injury to the neck resulting in fracture of the cervical vertebrae.

> Fractured or broken back where the lumbar vertebrae are fractured.

$>$ Injury to the spinal cord.
The damage to the spinal cord might be due to severing (total or partial), crushing/compression, or over-stretching/tearing. It is not the same as having a "broken back/neck" as this refers to a fracture of one or more of the vertebrae (bones that make up the spine), which does not necessarily lead to spinal cord injury.

PhysioFunction's Neurological Physiotherapy treatment is aimed at addressing the problems that can occur following SCI so that an individual can maximise their potential for recovery and therefore maximise their independence.

10. Whether the personal injury to that individual has resulted in quadriplegia/tetraplegia or paraplegia, treatment should be focused on what their needs are. Once the physiotherapist has made their assessment, and the individual has expressed what they would like to achieve, an appropriate course of physiotherapy can be agreed which will aid an overall rehabilitation The traumatic, and even more the non-traumatic paraplegia, always remaining a diagnostic and clinical challenge, has been a part of medical discussions and publications ever since quotations become an unavoidable element of the academic writings. In his prolific and pioneering work, Graves dedicated an entire lecture to the issue of paralysis, though he explicitly chose the term ,paraplegia ${ }^{\text {ee }}$ as its running head[1]. At that remote time of the 19th century, finding out that nervous injuries could follow a centripetal trajectory was highly sensitive, as Graves wrote: "When a certain portion of the extreme branches of the nervous tree has suffered an injury, the lesion is not confined merely to the part injured, but in many 
instances is propagated back towards the nervous centers" After marshalling several arguments that mirrored the general medical opinion of that time, paraplegia occurred due to bowel inflammation or enteritis. Nevertheless, Graves accepted that arsenical or lead poisoning might quite well be an etiological factor, with both the irritants acting directly on the central nervous system

11. Extensively illustrated and easy to use, this practical resource offers clear guidelines and stepby-step sequences for moving and working with individuals with differing levels ofparalysis. It serves as both an ideal student textbook and a valuable clinical manual for therapists who see tetraplegic and paraplegic patients.

12. The ability to accurately predict the magnitude of neurorecovery and expected functional outcomes after spinal cord injury is of great importance. This information is needed to justify medical and rehabilitation interventions to third party payers as well as to begin the process of planning for post discharge care. Over the past several decades, significant progress has been made in accurately predicting neurorecovery and its impact on functional outcomes.

\section{Method \& material:-}

This study included 25 patients according to the different age groups. Patients were analyzed based on clinical survey.

\section{Discussion:-}

SCI is an injury of the spinal cord which ranges from foramen magnum to the caudaequine. In response to the "primary damage" caused to the patient, some defense mechanisms, such as hemorrhage, inflammation and release of various chemicals, take place which lead to "secondary damage" The severity of the SCI depends upon the extent as well as the level of injury resulting in barriers to self dependence in terms of physical, functional, psychosocial and economic status.

The site of injury and region affected after SCI might cause tetraplegia or paraplegia. Tetraplegia refers to impaired functioning of the arms, trunk, legs and pelvic organs, whereas, paraplegia affects the trunk, legs and pelvic organs Besides this, many complications originate due to the hindrance in the normal activity such as improper bladder and bowel movement, urinary tract infections, pressure ulcers, orthostatic hypotension, fractures, deep vein thrombosis, spasticity, autonomic dysreflexia, pulmonary and cardiovascular problems, and depressive disorders. However, immobilization of the joints is one of the most common complications faced by patients with SCI The major cause of immobilization is the spasticity which occurs in about $70 \%$ of patients with SCI. It is also associated with the muscle paralysis, reduced muscle tone and absent tendon reflexes below the level of injury

Though, the treatment/management via pharmacological interventions plays a major role in the rehabilitation of patients with $\mathrm{SCI}$, a support through non-pharmacological measures is also important. An interdisciplinary approach is applied for the rehabilitation of patients with SCI, which includes a team consisting of clinicians skilled in hESC therapy and physiotherapists.

The present study focused on the role of physiotherapy in improving the mobilization in patients with SCI receiving hESC therapy. It reveals an improvement in mobilization in patients with chronic SCI after receiving a combination of hESC and physical therapy. The physical therapy aided in training of cells and took care of atrophy of limbs, whereas hESC therapy resulted in an overall improvement of the patients. This has been observed due to the reduction in the orthotic devices and use of mobility aids. A previous study also showed remarkable improvement in the clinical, locomotive as well as functional symptoms of the patients, where, $81.72 \%$ were able to walk with the support of calipers and mobility aids after receiving hESC therapy

Orthosis are mechanical, externally applied devices which are fit to the body parts for the restoration and maintenance of their anatomical and functional position According to International Standards Organization (ISO), they are used to modify the structural and functional characteristics of the neuromuscular and skeletal system The functional and regional classification of orthosis has been explained in 
International Journal of Trend in Scientific Research and Development (IJTSRD) ISSN: 2456-6470

\begin{tabular}{|c|c|c|c|c|c|c|c|}
\hline & $\begin{array}{c}\text { Cervical } \\
\text { orthosis } \\
(\mathrm{CO})\end{array}$ & $\begin{array}{c}\text { Head cervical } \\
\text { orthosis } \\
\text { (HCO) }\end{array}$ & & $\begin{array}{l}\text { adcervical } \\
\text { acic orthosis } \\
\text { (HCTO) }\end{array}$ & $\begin{array}{c}\text { Sacral } \\
\text { orthosis } \\
\text { (SO) }\end{array}$ & $\begin{array}{c}\text { Lumbo-sacral } \\
\text { orthosis } \\
\text { (LSO) }\end{array}$ & $\begin{array}{l}\text { Thoraco Lum } \\
\text { sacral ortho } \\
\text { (TLSO) }\end{array}$ \\
\hline \multirow{5}{*}{$\begin{array}{l}\text { REGIONAI } \\
\text { ORIHOSIS }\end{array}$} & \multicolumn{4}{|c|}{ Upper extremity orthosis } & \multicolumn{3}{|c|}{ Lower extremity orthosis (CALIPERS) } \\
\hline & \multicolumn{3}{|c|}{$\begin{array}{l}\text {-Shoulder and arm orthosis } \\
\text {-Elbow orthosis } \\
\text {-Wrist orthosis } \\
\text {-Hand orthosis }\end{array}$} & & \multicolumn{3}{|c|}{$\begin{array}{l}\text {-Foot orthosis (FO) } \\
\text {-Ankle-foot orthosis (AFO) } \\
\text {-Knee-ankle foot orthosis (KAFO) } \\
\text {-Hip-knee-ankle-foot orthosis (HKAFO }\end{array}$} \\
\hline & \multicolumn{3}{|c|}{ Supportive } & \multicolumn{4}{|c|}{ - Calipers, gaiters } \\
\hline & \multicolumn{3}{|c|}{ Functional } & \multicolumn{4}{|c|}{ - Orthotics for foot drop splint } \\
\hline & \multicolumn{3}{|c|}{ Corrective } & \multicolumn{4}{|c|}{ - Club foot boot in congenital talipes equino varus } \\
\hline \multirow{4}{*}{$\begin{array}{l}\text { FUNCTIONAI. } \\
\text { ORIHOSIS }\end{array}$} & \multicolumn{3}{|c|}{ Protective } & \multicolumn{4}{|c|}{ - Rigid four post collar for fractured cervical vertebrae } \\
\hline & \multicolumn{3}{|c|}{ Strengthening } & \multicolumn{4}{|c|}{ - In case of Tenodesis splint } \\
\hline & \multicolumn{3}{|c|}{ Pain relieving } & \multicolumn{4}{|c|}{ - Lumbosacral corset in case of pain in lower back } \\
\hline & \multicolumn{3}{|c|}{ Weight relieving } & \multicolumn{4}{|c|}{ - Pain relieving orthosis } \\
\hline
\end{tabular}

\section{Dia:-Classification of regional and functional orthosis}

Designing of a well fit orthotic device is important as it serves to be beneficial to patients with SCI undergoing chronic stage ambulation, thereby enhancing the therapeutic effect. Therefore, customized orthotic devices differ from the standard devices and are customized as per the need of the patient. The aim of physiotherapy should focus to make the patient independent as far as possible. To achieve this, strengthening of the weaker muscles rather than just supporting them should be the major goal. Moreover, an individual assessment should be performed as per patient ${ }^{\text {ee }} \mathrm{s}$ condition and deformity to customize the mobilization therapy.

The devices used for some of the patients are also customized, sometimes designed and manufactured by our centre. They are used by patients for standing upright, balancing, standing and walking. Besides mobilization, they limit/remove the risk of deformities, overstretching and contractures, as well as maintain the anatomical/functional position of the extremity. Studies also report the advantages of orthosis for primary management of SCI which involve the benefits associated with standing, walking for short distances, etc.

Calipers are the orthotic devices used in SCI cases with injury at T6-T9. The classification of calipers has been mentioned in Fig. They are fitted to the lower limb of SCI patients with the help of metal rods. KAFO is a type of orthotic device which stabilizes knee, ankle and foot, where the knee is supported by a caliper. In some cases, the joint is locked using a drop lock, spring loaded lock, cam lock, ball lock, dial lock or a plunger type of lock. The KAFOs are mainly used to support the patients with muscle weakness, upper motor neuron lesions and lost structural integrity However, combination of KAFO and a Shannon's brace provides better support. Shannon "s brace is used separately or combined with KAFO to provide waist support, once the patient shows improvement. It helps the patients in restoring his functional abilities. In context to locomotion, it helps a patient to become independent of supportive/mobility aids. 
The support to the weak musculature around the ankle joint is provided by AFOs. AFOs also help in gait exercise by stabilizing the joint for effective push-off during late stance, preventing toe-drag during swing. They also minimize the risk of falling as well as enhance the walking ability by providing safe joint mechanics. The AFOs should be designed support the ankles only and should not provide support to the calf muscles.

The improvement in the balance control, sitting and standing postures observed after the treatment with hESC therapy lead to a modification/alteration in devices. The custom made orthotic devices are modified by altering their support system, shape or size. Such a modification approach might help in improving the ability of the patients with SCI rather than in modifying their environment.

\section{MANAGEMENT OF PARAPLEGIA:}

\section{General Management:}

Frequent change of the patient ${ }^{\text {ee }}$ s posture to guard against bedsores.

$>$ Care of the skin by frequent washing with alcohol followed by talc powder. In case of urinary incontinence, frequent change of bed-sheets.

$>$ Care of the bladder: If there is retention, use parasympathomimetic drugs. If this fails, use a catheter to evacuate the bladder.

\section{Physiotherapy Management:}

1. Stretching/Flexibility Exercises:

$>$ slow, sustained lengthening of the muscle

$>$ Stretching is the most important exercise you can do.

$>$ Stretching improves flexibility - the ability to move the parts of your body through their full range of motion.

> Stretching also can reduce muscle spasticity and cramps and may also reduce problems such as tendonitis and bursitis.

$>$ To be effective, stretching routines must be done regularly, usually once or twice a day.

$>$ Stretch as far as you can and hold the stretch for 10 seconds and then ease back.

$>$ Each stretch should be performed slowly, with no sudden jerking or bouncing.

$>$ Stretching also should be done before and after other exercises to prevent muscle strain and soreness and to help avoid injuries.

2. Aerobic Exercises:

$>$ Steady exercise using large muscle groups
Aerobic exercise strengthens your heart and lungs and improves your body ${ }^{\text {ee }}$ ability to use oxygen. It also reduces fatigue, increases energy levels and helps you sleep better, control your weight, and lift your spirits.

$>$ It is generally recommended to gradually work up to three or four sessions per week, each lasting 15 to 60 minutes. Include a 5-minute warm-up (including stretching) before the activity and 5 to 10 minutes of a cool down (stretching and slower activity) afterwards.

$>$ Walking, stationary bicycling, water exercises and chair exercises are excellent choices.

\section{Walking:}

Experts recommend walking according to your ability, comfort and safety. Even short, slow walks can provide benefit. Aquatic (water) exercises: Aquatic exercises and swimming provide optimal exercise conditions. Water eliminates the effects of gravity, allowing weakened limbs to attain a greater range of motion. Water also helps support the body so there is less stress on hips, knees, and spine.

Exercises in the water: Can help increase muscle power and endurance and help mobilize joints and muscles. They also help to relax muscles and improve coordination. Warm water (between 83 and 90 degrees F) can be especially good for stiff, sore joints. Exercises can be done while standing in shoulderheight water or while sitting in shallow water. In deeper water, an inflatable tube, floatation vest or belt can be used for flotation.

Stationary bicycling: Stationary bicycling is a great way to improve fitness without putting stress on hips, knees, and feet. It can be done in any weather, and balance is generally not an issue. Add resistance only as you are comfortable and only after warming up.

Chair exercises: If mobility and balance are big issues, consider chair exercises. They can provide a great workout and easily incorporate strengthening and stretching exercises.

\section{Strengthening Exercises:}

$>$ Repeated muscle contractions until the muscle becomes tired.

Strengthening exercises help increase muscle tone and improve the quality of muscles. This enhances mobility and provides energy and a positive sense of well-being. 
Strong hip and leg muscles are needed to lift the legs to walk, and strong arm muscles are needed to carry out daily functions. Strong abdominal and back muscles help maintain correct posture and can counter pain resulting from poor gait, poor posture or the use of mobility aids.

\section{Physical agents:}

$>$ Thermotherapy: are used to decrease the pain and spasticity. They are (i) Superficial heat: IRR, wax bath, etc. (ii) Deep heat: SWD, MWD, etc.

$>$ Electrical therapy: is used to increase muscle power and to decrease the pain. e.g., TENS, EST, IFT.

\section{Therapeutic exercises:}

$>$ Mat exercise.

$>$ PNF exercise.

$>$ Active and passive ROM exercise.

$>$ Strengthening exercise.

$>$ Stretching exercise.

$>$ Endurance exercise

$>$ Co-ordination exercise.

$>$ Pelvic tilting exercise.

$>$ Hamstring muscle stretching.

$>$ Spinal rotation.

$>$ Calf muscle stretching.

$>$ Neck raising exercise

$>$ Knee rolling exercise.

$>$ Lying in extension.

$>$ Extension exercise.

$>$ Back and gluteal exercise.

\section{Symptomatic Treatment:}

$>$ Analgesics and sedatives for pain.

$>$ Muscle relaxants for the spasticity.

$>$ Vitamins and tonics

\section{Orthosis:}

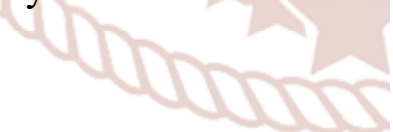

$>$ Various orthosis are used to assist patient with paraplegia.

$>$ These are: crutch, walker, cane, brace and wheelchair.

\section{Gait training:}

It is the important part of rehabilitation program balance can be achieved by proper gait training. Gait training can be done by following methods:

$>$ Pre ambulation MAT program:

$>$ Rolling, prone on elbow, prone on hand, quadruped, pelvic tilting, setting and standing balance.
$>$ Parallel bar progression

$>$ Advanced parallel bar activities.

$>$ Assistive device: E.g., Cane, crutches, walker

\section{Home program and Ergonomics:}

$>$ Patient is advised to use the lumbosacral orthosis to support the back during traveling.

Patient is advice for hot fomentation at home.

$>$ Patient is advised to lying in prone position for at least 15 minutes duration twice in a day.

$>$ Patient is explained about the proper sitting, standing, lying and lying to standing, doing the household activity in a proper way.

$>$ Patient is advised to take rest and to avoid the forward bending as much as the patient can avoid.

\section{Result:-}

The physiotherapists, with the help of the clinicians, measured the level of improvement in the patients

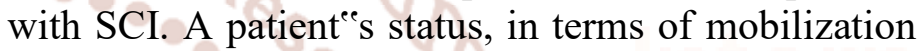
and improvement, was to assess the progress in posture, balancing, standing, sitting, walking, etc. The patients were assessed with AIS impairment scale for the assessment of improvement in patients.

\section{No. of Sample size:- 25}

Before treatment graph will be:-

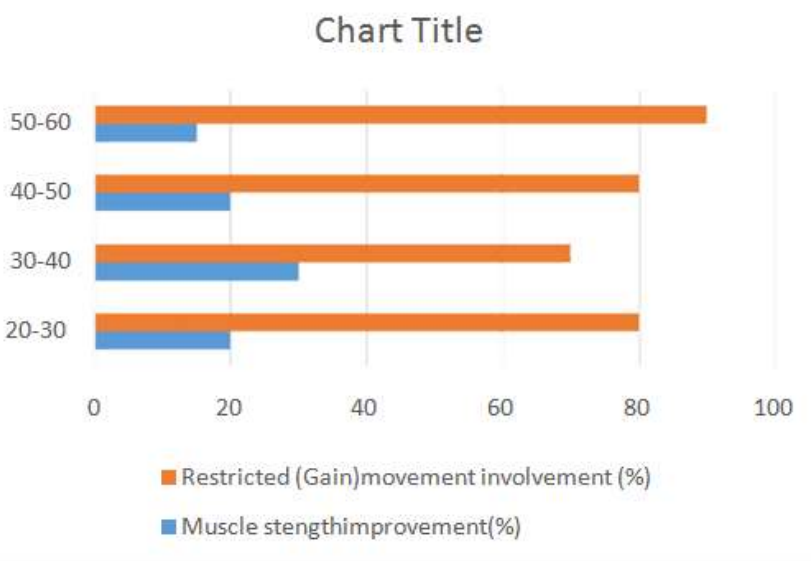

After Physiotherapist treatment:Chart Title

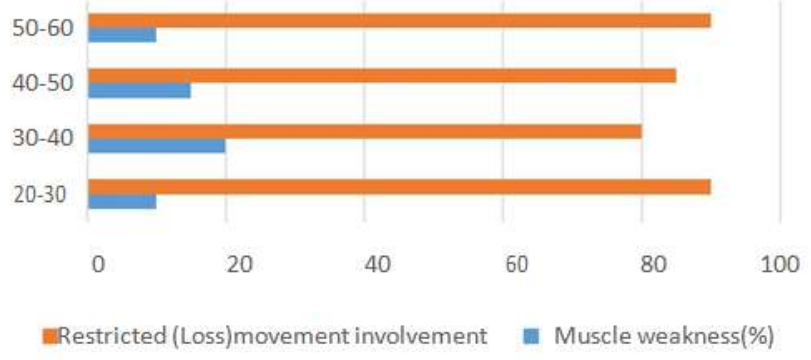




\section{Conclusion:-}

Reduction of pain and swelling, improvement in motion, improvement in gross and fine motor skills, and relaxation and healing of soft tissues is the main focus of physiotherapy. Still, it helps the patients to live with their disabilities. The regenerative capacity of SCI translating into improvements in quality of life in patients with chronic SCI seems to be very encouraging. The HESC therapy along with physiotherapy which addresses the regeneration that is going on in the patient could herald a new approach in the treatment of SCI.

\section{Reference:-}

1. Www.portea.com

2. www.portea.com

3. https://jamanetwork.com

4. https://sites.google.com

5. Somers, MF: Spinal Cord Injury: Functional Rehabilitation, ed 2. Prentice-Hall, Upper Saddle River, NJ, 2001
6. National spinal card injury Statistical center: Spinal cord Injury: Facts and Figures at a Glance. August 2004. National Spinal Cord Injury Statistical Center. University of Alabama, Birmingham, AL 35249-7330. Retrieved March 12, 2006 from http://www.spinalcord.uab.edu/shw.asp?durki=21 446

7. https://www.ncbi.nlm.inh.gov

8. www.physio-pedia.com

9. www.epainasssist.com

10. www.physiofnction.co.uk

11. Bromley, I: Tetraplegia and Paraplegia, ed 3. Churchill Livingston New York, 1985

12. Nixon, V: Spinal Cord Injury: A Guide to Functional Outcomes in Physical Therapy Management. Aspen, Rockville, MD, 1985

13. www.sciencedirect.com 